\title{
The activity of LE10 peptide on biological membranes using molecular dynamics, in vitro and in vivo studies
}

\author{
Egipto Antunes ${ }^{\mathrm{a}}$, Nuno G. Azoia ${ }^{\mathrm{a}}$, Teresa Matamáa ${ }^{\mathrm{a}, \mathrm{b}}$, Andreia C. Gomes ${ }^{\mathrm{b}}$, Artur Cavaco-Paulo ${ }^{\mathrm{a}, *}$ \\ a Biological Engineering Department, University of Minho, Campus of Gualtar, 4710-057 Braga, Portugal \\ ${ }^{\mathrm{b}}$ Molecular and Environmental Biology Centre (CBMA), Department of Biology, University of Minho, Campus of Gualtar, 4710-057 Braga, Portugal
}

\section{A R T I C L E I N F O}

\section{Article history:}

Received 6 September 2012

Received in revised form 16 January 2013

Accepted 17 January 2013

Available online 4 February 2013

\section{Keywords:}

Antimicrobial peptides

Cell-penetrating peptides

Liposome disruption

Membrane active peptides

Molecular dynamics

\begin{abstract}
A B S T R A C T
Cell-penetrating peptides (CPPs) and antimicrobial peptides (AMPs) are generally defined as small cationic peptides with the ability to interact with lipidic membranes, in a process driven by electrostatic and hydrophobic processes. The interaction with CPPs is known to lead to its translocation across the membrane, while with AMPs lead to membrane damage.

Here we present one synthetic anionic peptide, LE10 (LELELELELELELELELELE), which strongly interacts with model membranes, showing properties of CPPs (translocation through lipidic membranes on a mechanism usually described for cationic CPPs) and AMPs (membrane disruption) in molecular dynamic studies, experimental studies with liposomes and mammalian cells in vitro.

Based on the LE10 properties here demonstrated, small modifications in its structure could make it a very promising tool for drug delivery.
\end{abstract}

(C) 2013 Elsevier B.V. All rights reserved.

\section{Introduction}

Cell-penetrating peptides (CPPs) and antimicrobial peptides (AMPs) are two groups of short peptides with the ability to interact with cell membranes with different outcomes.

CPPs are molecules that rapidly internalize across cell membranes and can transport macromolecules into living cells [1]. These molecules are, generally, short peptides (up to 30 amino acids) with positive net charge (rich in lysines and/or arginines) [2]. The mechanism of translocation of the membrane by CPPs remains unclear, and three main entry mechanisms have been proposed in the literature, namely direct translocation [3], different forms of endocytosis, such as macropinocytosis [4], clathrin-mediated endocytosis [5] and caveolae/lipid raft-mediated endocytosis [6], and penetration through a transitory structure, like micelles [7] and pores [8].

AMPs, in turn, are antibiotic molecules, having the ability to kill a broad spectrum of microorganisms and cells, like bacteria, parasites, tumor cells, fungi and viruses $[9,10]$. In most cases, AMPs are cationic, and interact with the target membrane surface, in a process involving interactions between the charged residues of the peptides and anionic components of the cell membrane surface [10], killing the target by membrane disruption or permeabilization, or translocating membrane and killing the target by other mechanisms. Some of these mechanisms are inhibition of DNA

\footnotetext{
* Corresponding author. Tel.: +351 253604400; fax: +351 253604429

E-mail address: artur@deb.uminho.pt (A. Cavaco-Paulo).
}

or RNA synthesis, blocking of chaperones, inhibition of ribosomal activity, and induction of reactive oxygen species or disruption of mitochondrial cell membrane [11].

Unlike CPPs, the existence of negatively charged AMPs is well established, and this type of AMPs has been known since 1980 [12]. Thus, according to their charge, AMPs can be classified as cationic AMPs (CAMPs), generally with a net charge in the range of +4 to +6 due to presence of lysine and arginine, or as anionic AMPs (AAMPs), normally with a net charge in the range of -1 to -7 , due to glutamic and aspartic acid residues.

Although AAMPs have been described in a wide range of organisms, from vertebrates to plants [12], the number of studies with these peptides is very low, compared with CAMPs, and their method of action is still unclear.

On the other hand many models of CAMPs membrane activity have been proposed. The most common are the barrel stave pore model [13], the toroidal pore model [14], the carpet model [15] and the detergent model [16].

To our knowledge there is only one anionic CPP (ACPP) described, the "SAP(E)" (VELPPPVELPPPVELPPP). The SAP(E) is a synthetic peptide and results of one investigation about the role of peptides net charge in its cell-penetrating properties [17]. Starting from a well-known CPP, SAP (VRLPPPVRLPPPVRLPPP), the authors changed the positive arginine by glutamic acids. The results clearly show that the positive net charge is not a fundamental feature to the SAP activity, since the SAP(E) showed similar uptake efficiency and mechanism of action. It is expected that this fact may also be true for other CPPs. 
CPPs are widely studied to be used in drug delivery and gene therapy as they can translocate cell membranes transporting cargos. In turn, due to their antimicrobial activity and the processes involved, the AMPs have been studied as potential new antibiotics. Some authors explore the hypothesis that these two types of molecules are in fact one, because of their similarity in structure and properties [18-20]. Both share some mechanisms of membrane interactions, and there are reported cases of CPPs with antimicrobial activity, like the TAT [21-23] and penetratin [24,25] peptides, as well as AMPs that penetrate host cells without damaging their membrane, like Bac7 [26] peptide. This unifying class of peptides was designated as membrane-active peptides (MAPs) [19].

More research is needed in this field of membrane-active peptides to determine if AMPs and CPPs are two independent groups or not. Better elucidation of the mechanisms involved in the interaction with their targets, and the features of their composition and structure that are necessary to their activity are also unknown.

In this work, we report one synthetic peptide, LE10 (LELELELELELELELELELE) that is short and negatively charged. This peptide exhibits membrane activity in molecular dynamics and experimental studies, leading to membrane bending, liposome disruption and mammalian cell death. A series of molecular dynamic studies were performed to elucidate the interaction of the peptide model with lipidic membranes models (see Table 1 in Section 2 for an overview of the molecular dynamics simulations that were performed). Then dynamic light scattering, fluorescence spectroscopy and fluorescence microscopy studies were done, using liposomes with, approximately, the same composition of the membrane models used in the simulations, and LE10 peptide, to validate the computational results. Finally the effect of the peptide in vitro was studied, using one human cell-line and six microorganisms (four bacteria and two yeasts). This peptide display features of both AMPs (leading to cell dead) and CPPs (penetration into the membrane). These intriguing results may help to elucidate some aspects about the mechanism of interaction of anionic membrane active peptides. Cell penetrating properties of LE10, here demonstrated, make it a very promising tool for the development of new drug-delivery vectors.

\section{Material and methods}

\subsection{Molecular dynamics simulations}

An overview of the simulations performed is presented in Table 1. The peptide model was created with the software Pymol 1.1 [27], and the software CELLmicrocosmos 2.2 [28] was used for create all membrane models. The lipidic bilayers of 1-palmitoyl2-oleoyl-phosphatidylcholine (POPC, $\mathrm{C}_{42} \mathrm{H}_{82} \mathrm{NO}_{8} \mathrm{P}$ ) and cholesterol $\left(\mathrm{CHOL}, \mathrm{C}_{27} \mathrm{H}_{46} \mathrm{O}\right.$ ) have a proportion (mol:mol) of $60-40 \%$, respectively. All molecular dynamics simulations were performed using the Gromacs 4.0.7 package [29], with GROMOS96 43a1-S3 force field [30], with periodic boundary conditions, at constant temperature $(300 \mathrm{~K})$ and pressure ( $1 \mathrm{bar})$. Detailed information about the simulations is presented as Supplementary material.

A few simulations were performed for each system, with different starting velocities taken from a Maxwell distribution. Initially some simulations were done with peptide and bilayers alone, to equilibrate both structures. The equilibrated structures were used to perform the interaction simulations. In these simulations the peptide(s) was(were) added to the water layer of the membrane system (see Fig. 1).

\subsection{Liposomes assays}

The LE10 peptide was purchased from GenScript USA Inc. (NJ, USA). Phosphatidyl choline from egg lecithin (EPC) was purchased from Lipoid (Germany), CHOL $\left(\mathrm{C}_{27} \mathrm{H}_{46} \mathrm{O}\right)$ from Sigma (Spain), and Nile Red $\left(\mathrm{C} 2 \mathrm{OH} 18 \mathrm{~N}_{2} \mathrm{O}_{2}\right)$ from Invitrogen (Spain). The size and polydispersity index (PID) were measured at $25^{\circ} \mathrm{C}$, with a Zetasizer Nano ZS from Malvern (England), which uses dynamic light scattering and the Stokes-Einstein relationship, with a cumulant analysis (according the ISO standard document 13321:1996 E) [31], to calculate these features. Fluorescence kinetic measurements were carried on a Synergy Mx spectrofluorimeter from BioTek (Germany). The measurement of Nile Red fluorescence was made at $25^{\circ} \mathrm{C}$, during $3 \mathrm{~h}$, with an emission wavelength of $620 \mathrm{~nm}$ using $495 \mathrm{~nm}$ as the excitation wavelength. The visualization of liposomes was made using a Leica Microsystems DM5000 B epifluorescence microscope equipped with a $100 \mathrm{~W} \mathrm{Hg}$ lamp and an appropriate filter setting, for $25 \mathrm{~min}$. Digital images were acquired with Leica DFC350 FX digital Camera and Leica Microsystems LAS AF software, version 2.0 (Leica Microsystems GmbH, Wetzlar, Germany).

\subsubsection{Liposomes preparation}

All liposomes were prepared with Nile Red, EPC and CHOL in the molar proportion of 60\% EPC and 40\% CHOL. They were prepared by lipid film hydration method, with a final concentration of $3000 \mu \mathrm{M}$ of lipids, and $3 \mu \mathrm{M}$ of Nile Red. Chloroform (98.9\%) was used to dissolve EPC and CHOL lipids, and the solution was evaporated in a rotary evaporator, for approximately $2 \mathrm{~h}$, and then kept under high vacuum to full evaporate the solvent for another $2 \mathrm{~h}$. The obtained lipid film was allowed to hydrate in water $(15 \mathrm{~mL})$ with vigorous stirring and with 1-2 s of ultrasonic bath, resulting in multillamelar vesicles dispersion. In order to obtain one population of liposomes big enough to be observed in a fluorescence microscope, the dispersion obtained directly from the hydration process was used. To avoid nonspecific fluorescence, and other fluorescence problems, this dispersion was filtered through a filtration column (PD-10 Desalting Column containing of Spephadex TM $(8.3 \mathrm{~mL})$ from GE Healthcare) to obtain one population of vesicles without Nile Red in supernatant solution. This dispersion was only used for the observation of the liposomes under microscope. For obtaining a homogeneous population of liposomes, the obtained dispersion of multillamelar vesicles was extruded about 30 times

\section{Table 1}

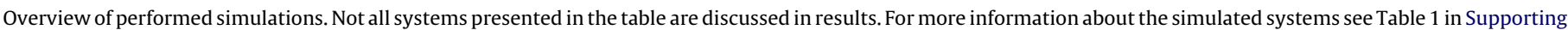
information document.

\begin{tabular}{|c|c|c|}
\hline Label & System & No. of constituents \\
\hline LE10-W & Peptide in water & LE10: 1 ; waters: 15,828 \\
\hline POPC-CHOL-S & Small bilayer & POPC: 124 ; CHOL: 84 ; waters: 17,524 \\
\hline POPC-CHOL-M & Medium bilayer & POPC: 460 ; CHOL: 308 ; waters: 47,286 \\
\hline POPC & Medium bilayer without $\mathrm{CHOL}$ & POPC: 266 ; waters: 16,913 \\
\hline POPC-CHOL-LE10-S & Small bilayer with peptide & POPC: 124 ; CHOL: 84 ; LE10: 1 ; waters: 16,797 \\
\hline POPC-CHOL-LE10-M & Medium bilayer with peptide & POPC: 460; CHOL: 308; LE10: $1-4$; waters: $32,000-35,566$ \\
\hline POPC-LE10 & Medium bilayer without CHOL with peptide & POPC: 266 ; LE10: $1-3$; waters: $16,763-16,496$ \\
\hline POPC-CHOL-LE10-L & Large bilayer with peptide & POPC: 1344 ; CHOL: 897 ; LE10: 1 and 4 ; waters: 185,891 \\
\hline
\end{tabular}




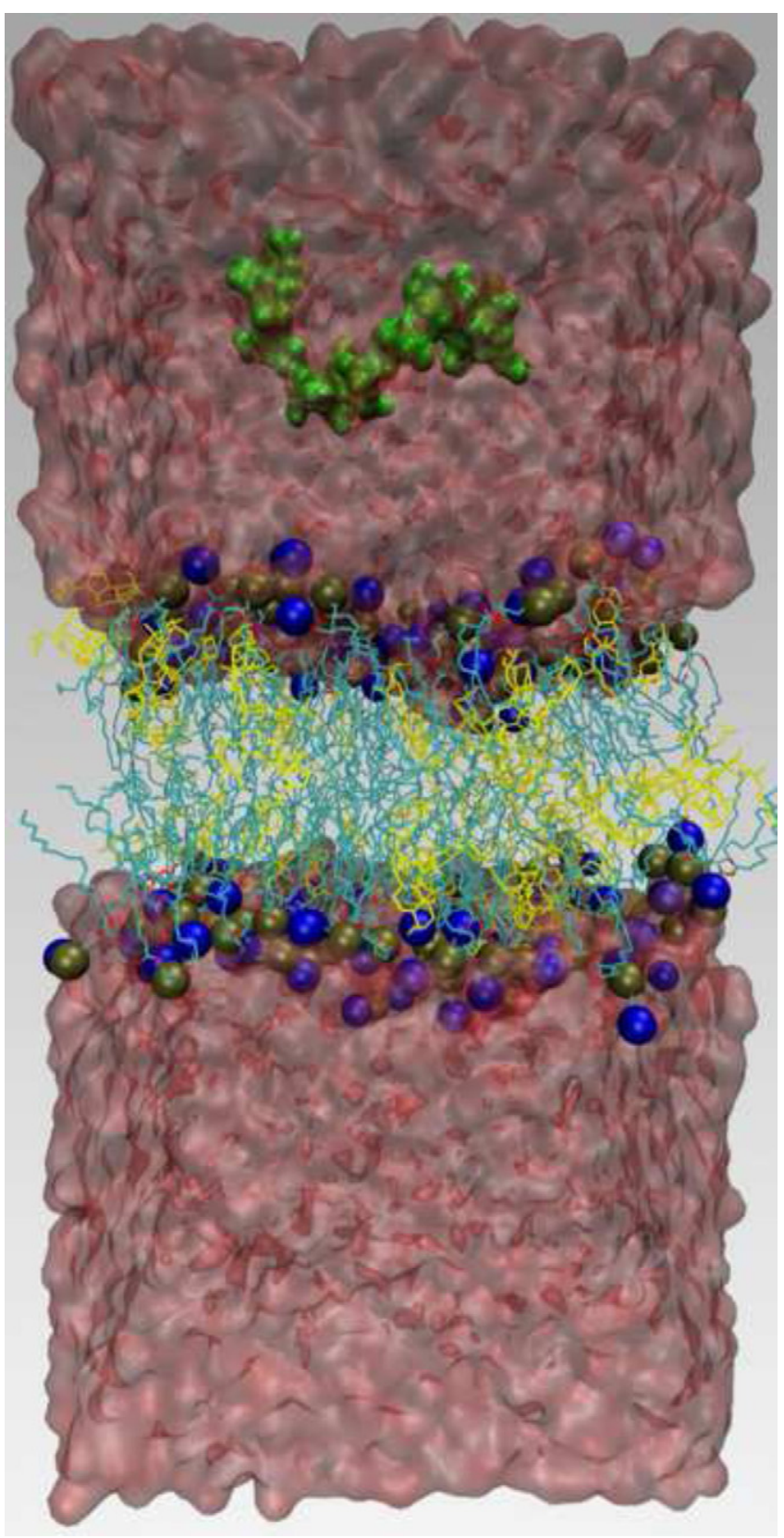

Fig. 1. Snapshot of the starting configuration of a system POPC-CHOL-S-LE10. The water is represented as translucent surface (red), the peptide as surface (green), the POPC headgroups as blue (choline) and brown (phosphate) spheres. CHOL (yellow) and lipid tails of POPC (cyan) are sticks. (For interpretation of the references to color in this figure legend, the reader is referred to the web version of the article.)

through polycarbonate membranes having a $100 \mathrm{~nm}$ pore diameter (in an Avanti Mini-Extruder). To check the features of interest (size, PDI, fluorescence and imaging), with or without incubation with the LE10 peptide, the different liposome solutions obtained previous were diluted from $3000 \mu \mathrm{M}$, take in account the necessary volume of peptide stock solution ( $300 \mu \mathrm{M}$ LE10) in water, to have the final concentration of 30 and $300 \mu \mathrm{M}$ of peptide and $500 \mu \mathrm{M}$ of liposomes in the resulting solution.

\subsection{In vitro assays}

The h-TERT-immortalized foreskin fibroblast human cell line BJ-5ta was purchased from ATCC through LGC Standards, and was maintained according to the supplier's recommendations. The medium was composed by a mixture 4:1 of DMEM (Dulbecco's Modified Eagle's Medium) and Medium 199, supplemented with fetal bovine serum (FBS, 10\% (v/v)), Hygromycin B (10 $\mu \mathrm{g} / \mathrm{mL})$ and Penicillin/Streptomycin antibiotic mixture $(1 \%(\mathrm{v} / \mathrm{v}))$. The dry powdered DMEM, containing L-glutamine $(4 \mathrm{mM})$ and glucose $(4.5 \mathrm{~g} / \mathrm{L})$, was previously prepared and sodium bicarbonate was added to a final concentration of $1.5 \mathrm{~g} / \mathrm{L}$. FBS was purchased from Lonza (Walkersville, Inc., MD, USA), the media and the other supplements were purchased from Sigma-Aldrich Chemie GmbH (Buchs, Switzerland). The cells were cultured at $37^{\circ} \mathrm{C}$ in $\mathrm{CO}_{2}$ atmosphere (5\%). The cells were incubated with the LE10 peptide one day after seeding 55000 cells/well in a 24-wells tissue culture plate (from TPP, Switzerland). The LE10 was solubilized in water and sterilized by filtration, then, it was diluted in culture medium to the final concentrations of 3 and $300 \mu \mathrm{M}$. Cells were incubated in duplicate with $500 \mu \mathrm{L}$ of each peptide dilution for $24 \mathrm{~h}$ at $37^{\circ} \mathrm{C}$ in $5 \%$ $\mathrm{CO}_{2}$ atmosphere. Cells incubated with dimethyl sulfoxide (DMSO, $30 \%$ ) were used as positive control and cells without any treatment were used as negative control for the cytotoxicity assays. The LE10 cytotoxicity was evaluated by lactate dehydrogenase (LDH) leakage to the culture medium as a measure of membrane integrity and by the MTT (3-(4,5-dimethylthiazol-2-yl)-2,5-diphenyltetrazolium bromide) reduction assay as a measure of cellular metabolic viability.

\subsubsection{MTT assay}

After one day of incubation with the peptide, MTT solution ( $50 \mu \mathrm{L}, 5 \mathrm{mg} / \mathrm{mL}$ ) was added to each culture well, and cells were incubated another $2 \mathrm{~h}$ at $37^{\circ} \mathrm{C}$ in $5 \% \mathrm{CO}_{2}$ atmosphere. The medium was discarded and DMSO/Ethanol mixture $(500 \mu \mathrm{L}, 1: 1 \mathrm{v} / \mathrm{v})$ was added. After complete MTT formazan solubilization, the resulting solution $(150 \mu \mathrm{L})$ was transferred to 96 -well plate and the absorbance was read at $570 \mathrm{~nm}$ and $690 \mathrm{~nm}$ in a multiwell plate reader spectrophotometer. The relative cell viability was calculated having the negative control absorbance as reference.

\subsubsection{LDH assay}

In order to determine extracellular LDH, the medium was collected and kept at $-80^{\circ} \mathrm{C}$ until quantification. The cells were lysed with Tris (hydroxymethyl) aminomethane (TRIS) buffer (15 mM); the lysate was collect and kept at $-80^{\circ} \mathrm{C}$ until quantification. The LDH activity was quantified by the decrease in NADH absorbance at $340 \mathrm{~nm}$ during $3 \mathrm{~min}$ at $30^{\circ} \mathrm{C}$, using a microplate reader. The final reaction volume $(300 \mu \mathrm{L})$ consisting in NADH $(250 \mu \mathrm{M})$ and pyruvate $(280 \mu \mathrm{M})$ in phosphate buffer $\mathrm{pH} 7.4(50 \mathrm{mM})$. To measure the intra and extracellular LDH activity, NHEM lysate $(45 \mu \mathrm{L})$ and culture medium $(90 \mu \mathrm{L})$ were used, respectively. The percentage of LDH-release was expressed as the relation between extracellular activity and total LDH activity.

\section{Results and discussion}

\subsection{Molecular dynamics}

The first system that was simulated for the study of the interaction of the LE10 peptide with the 1-Palmitoyl-2-oleoylphosphatidylcholine (POPC) and cholesterol (CHOL) bilayers was POPC-CHOL-LE10-S (see Table 1 for labels identification). The system consists in one small bilayer in water with one peptide (see Fig. 1).

Every replica of this first simulation showed that the peptide rapidly interacts with the bilayer surface and leads to membrane bending. With the buckling of the membrane, the interactions between periodic images became relevant and started to direct the evolution of the system, leading to artificial results. In this case, 


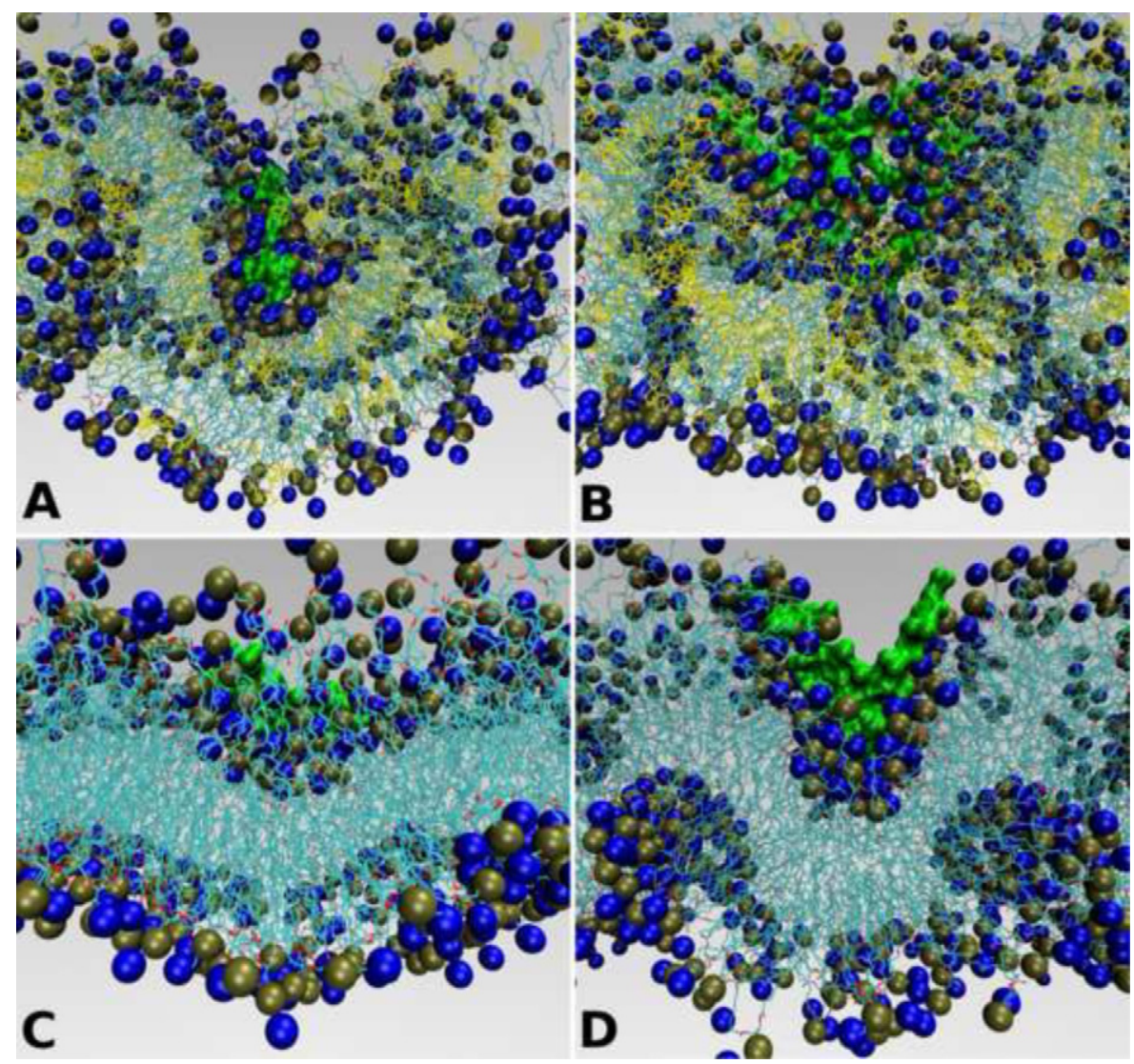

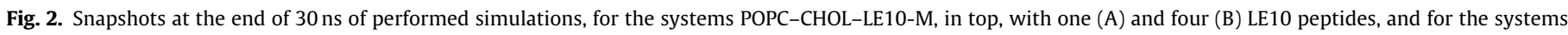

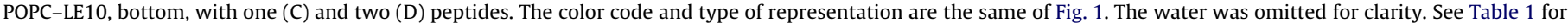
more information about the simulated systems.

these artifacts showed global destabilization of the lipidic bilayer (data not show).

Due to this problem, the lateral size of the system was increased, from about $7.5 \mathrm{~nm}$ to $14 \mathrm{~nm}$. In this bigger system (POPC-CHOL-LE10-M) the interaction of LE10 with the membrane showed that the peptide leads to membrane bending, as seen in previous simulations, but the membrane was not globally destabilized. Instead the peptide was involved by the bilayer (see Fig. $2 \mathrm{~A}$ and $\mathrm{B}$ ). This difference in the results is a consequence of the different size of the systems, showing that it is necessary to be careful with interactions between periodic images.

Despite the increase of the lateral size of the simulated bilayer, after tens of nanoseconds (approximately $20 \mathrm{~ns}$ for membranes with one and $15 \mathrm{~ns}$ with four peptides) the problem of lateral interactions with periodic copies reappears. This is due to the strong interaction between the peptide and the membrane, leading to the nearly full bending of the membrane. At the end of simulations, the membrane involves the peptide like one half of a micelle. This strong interaction is responsible for the decrease on the width of the simulation box, which makes the interactions between periodic images relevant again. These simulations also showed that the phenomenon of bending is concentration dependent. In different simulations with membranes of the same size, the number of LE10 peptides added altered both bending velocity and degree. The bending is faster and more intense with increasing peptide number (see Fig. 2A and $\mathrm{B}$ ).

The envelopment of the peptide by the bilayer, visualized in the previous simulations, raises the question if the peptide is internalized by the membrane, by micelle or vesicle formation, since the internalization of peptides by micelles is one of the mechanisms of internalization proposed in the literature for CPPs [7]. To answer this question, one bigger system was built, with a lateral size of $26 \mathrm{~nm}$ (POPC-CHOL-LE10-L). This system was also built to overcome the problems with periodic images. In Fig. 3 is represented the evolution of the simulated model membrane over time, with one or four peptides. With the simulation of these larger systems it becomes clear that, due to the interaction with the peptide, the membrane will evolve from flat lipid bilayer to a system where the bilayer presents a negative curvature, similar to half of a micelle, near to the peptide localization. This curved region of the membrane eventually coalesces into a vesicular bud or a micelle. In these simulations, it is also noticeable that the process of bending is concentration dependent, which was also suggested by previous simulations (see Fig. 3). Initially the interaction between the peptides and the membrane, for the system with four peptides, evolves very rapidly (few tens of nanoseconds) and the system seems then reach equilibrium. This equilibrium corresponds to the complete engulfment of the peptides by the membrane. The four peptides stay together throughout the simulations, interacting with each other and with the membrane. This aggregation of the peptides is a result of the interaction with the membrane. In independent simulations with four LE10 peptides in water (results not showed) it was not possible to observe peptide aggregation.

At this point, the reorientation of lipids to form one complete vesicle or micelle, and their fission from the membrane, will require a higher energy, since these processes have a high free energy threshold [32]. Short simulations do not reach the time scales necessary to evaluate complete vesicle creation and their fission from the membrane. Hyung-June Woo and Anders Wallqvist studied Magainin 2, by coarse grained simulations [33], and although they reach time scales in the order of microseconds, these authors did not observe the fission of vesicular buds formed. This confirms 


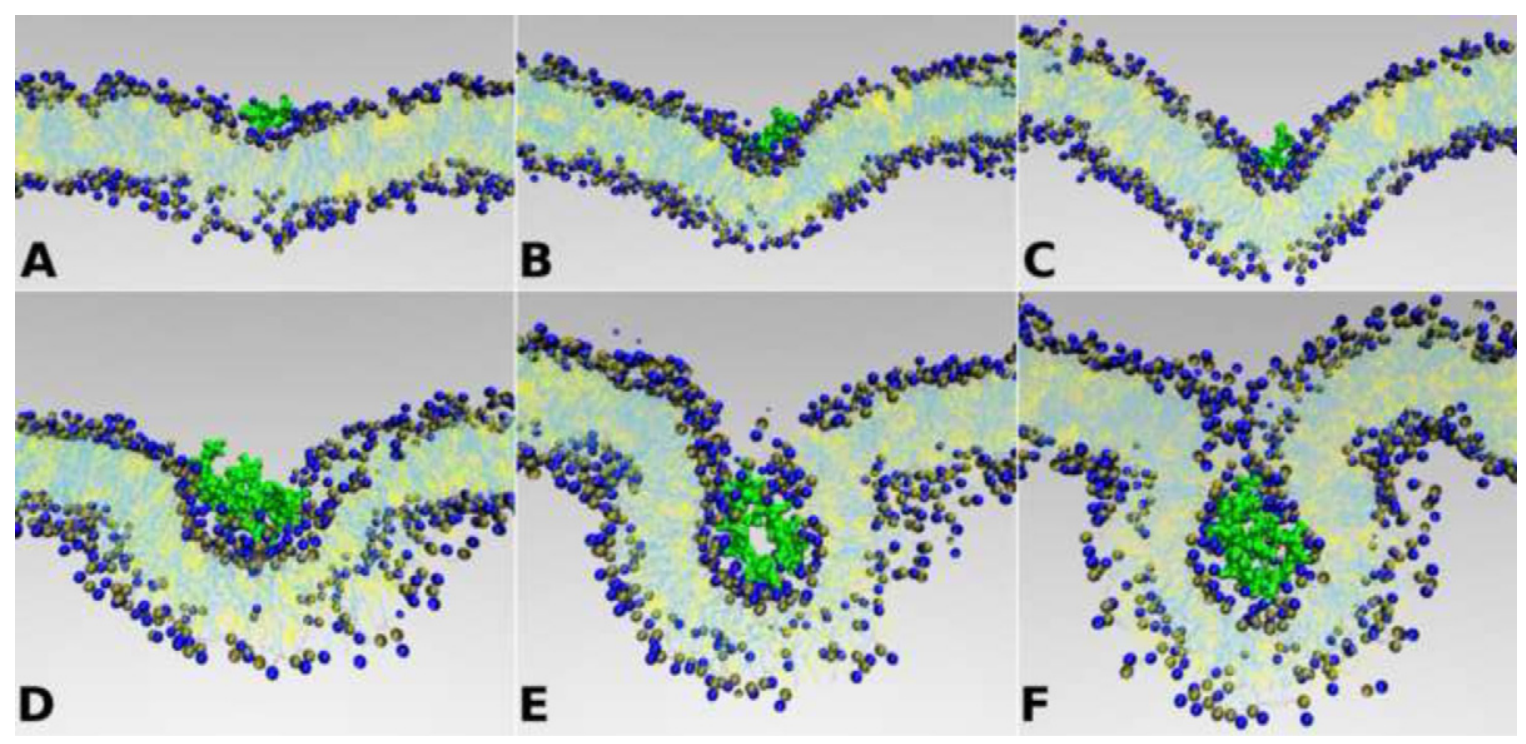

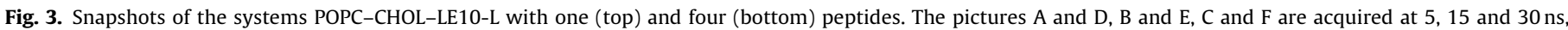

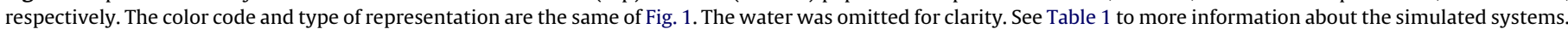

the difficulty to observe the vesicle fission from membranes in our molecular dynamics studies. Even if we take into account the differences between their and our systems.

The mechanism of interaction between peptide and lipids seems to be electrostatic. The negatively charged residues of glutamic acid are attracted by the positive charges of the choline head groups of POPC lipids, but the negatively charged phosphate groups repel these residues. These balance of opposing forces results on membrane deformation. Although the attraction between the choline groups and the glutamic acids is strong, and the LE10 have 50\% of hydrophobic content, the peptide was never able to penetrate the membrane. The peptide only interacts with the bilayer surface, which then involves the peptide.

To assess if the CHOL molecules have any role in this process, the behavior of the systems with one membrane without this steroid was also simulated (POPC-LE10). The lateral size of these POPC membranes was a little smaller than the previous bilayers of POPC and CHOL (POPC-CHOL-LE10-M). The same effect of membrane bending appears and remains concentration dependent (see Fig. $2 \mathrm{C}$ and $\mathrm{D}$ ), but the velocity and the degree of bending is lower than in simulations with CHOL. This can be due to the higher fluidity of membranes without CHOL [34] that could rearrange more easily in response to the stress induced by the presence of the peptide.

\section{Dynamic light scattering}

To determine if the behavior demonstrated by molecular dynamics studies actually occurs and is not an artifact, liposomes of phosphatidyl choline from egg lecithin (EPC) and CHOL were prepared, in a proportion of 60-40 (mol:mol), in order to study the interactions between the LE10 peptide and the membranes. Nile Red dye was also incorporated in the liposomes.

The size and PDI were measured, both for the liposomes and the liposomes-peptide systems over $24 \mathrm{~h}$ of incubation with LE10 (30 or $300 \mu \mathrm{M}$ ). The results clearly show that the peptide interacts with the lipidic membrane of the liposomes (Fig. 4). The lower concentration of peptide tested does not significantly affect the size of the liposomes, even after $24 \mathrm{~h}$ of exposure, while the PDI is lightly increased. With the higher peptide concentration tested, the liposomes were immediately affected, as indicated by the two measured parameters. The size measured for this dispersion at the beginning of incubation is significantly higher than the size measured for the liposomes and liposomes with lower concentration of LE10. For these measurements (with LE10 at $300 \mu \mathrm{M}$ ) it is possible to observe a big dispersion of the values read. This can be an indication of the inhomogeneity of the dispersion, caused by the peptide, although there seems to be an increase in the homogeneity at $24 \mathrm{~h}$ of incubation. The interaction of the peptide, at a concentration of $300 \mu \mathrm{M}$, with the liposomes is able to affect their size and the size distribution.

The results from the incubation experiments with the peptide LE10 gave some insights on the mechanisms of the interaction between this peptide and the membranes. The liposomes alone are stable during the period of incubation, but the liposomes incubated with the peptide display a different behavior depending on the concentration, which is in line with the results from the simulations, where it was possible to observe that the interaction is concentration dependent. For the lowest peptide concentration, the results showed that there is a slight increase in liposome size at the end of the experiments, but for the highest peptide concentration it is possible to observe a big increment on the size immediately after the

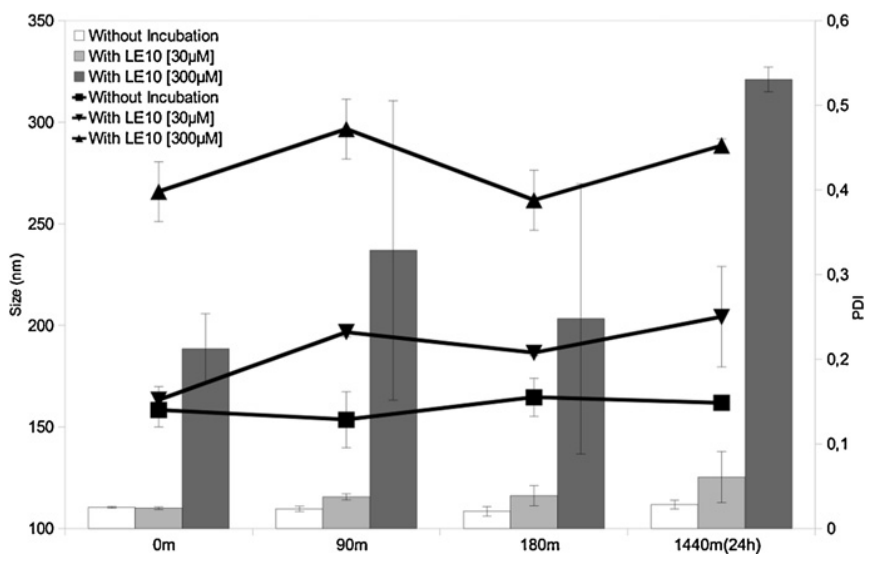

Fig. 4. Results of dynamic light scattering of liposomes of POPC and CHOL (60:40 mol:mol) with Nile Red, with and without incubation with the LE10 peptide, at $0,90,180 \mathrm{~m}$ and $24 \mathrm{~h}$. The columns indicate the Size and the lines the polydispersity index (PDI) values. Average values from two replicas for each condition and time point (the error bars represent the standard deviation of the replicas at 95\%). 
addition of the peptide. This size increment is even more evident at the end of $24 \mathrm{~h}$.

The most remarkable conclusion is that the incubation with the LE10 peptide dramatically changes the population of liposomes, since the high PDI values and deviation of average values do not allow a good characterization of the resulting population of particles. The differences and the high deviation of the values measured can be explained by liposome disruption and subsequent aggregation of the lipids in other structures, like disordered lipidic clusters, giant liposomes, micelles or vesicles, with very wide size distribution, due to random lipid rearrangement. These features measured by dynamic light scattering do not contradict the mechanisms evidenced in molecular dynamics simulations. In fact, they could even be explained by these mechanisms. High concentrations of the peptide may provoke the liposome disruption, due to a higher level of destabilization.

\subsection{Fluorescence spectroscopy and microscopy}

The results of dynamic light scattering clearly shows that the peptide interacts with the liposomes, but do not disclose the exact mechanism. The studies of fluorescence with Nile Red were done in an attempt to unveil this mechanism. Nile Red is a lipophilic probe, poorly soluble and fluorescence in water. This probe was added in the preparation of all liposomes, and due to the Nile Red lipophillicity, it is expected to be locate at the liposomes membranes. The fluorescence yield of this probe is enhanced in hydrophobic environments, and shifts in its maximum emission value of fluorescence are indicators of conversions of lipidic phases [35,36].

Fig. 5 represents the results of the fluorescence kinetic from Nile Red performed over $180 \mathrm{~min}$, with and without LE10 incubation. The fluorescence value is approximately the same for the three solutions at the beginning of the measurement. The incubation with LE10 makes the fluorescence value to increase over time, and the increment is concentration dependent. One again these results can be explained by the mechanism observed in the simulations, and by liposome disruption. The membrane invagination, and the

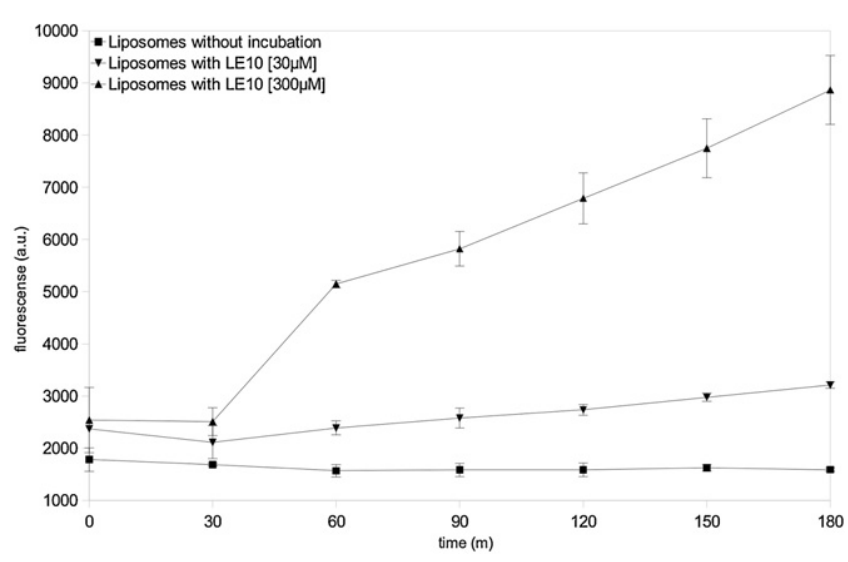

Fig. 5. Fluorescence kinetic $(620 \mathrm{~nm})$ over $180 \mathrm{~min}$ at $25^{\circ} \mathrm{C}$, from Nile Red in samples of liposomes with and without incubation with LE10 peptide. Average values from three replicas for each condition and time point (the error bars represent the standard deviation of the replicas at 95\%).

disruption of liposomes with subsequent rearrangement of the lipids in other structures, may provide more hydrophobic environments to Nile Red.

In order to confirm if the peptide leads to liposome disruption, samples of multilamellar liposomes, obtained directly from the hydration process without extrusion, were exposed to LE10 and observed in a fluorescence microscope. The size of these liposomes without incubation was between 2 and $5 \mu \mathrm{m}$ (measured in the Zeta Sizer). The time evolution of one sample of liposomes with Nile Red, incubated with LE10 $300 \mu \mathrm{M}$, was followed for $25 \mathrm{~min}$ (see Fig. 6). Initially, Fig. 6A and B, it is only possible to observe some red particles, with an approximate size of $7 \mu \mathrm{m}$, which may correspond to big vesicles, or vesicles aggregates. Over the time, huge structures, in the form of large aggregates and/or films become visible, and the previous small red particles begin to be less frequent (Fig. 6C and D). In the last pictures, the frequency of observed big structures increases, while the small red particles were barely

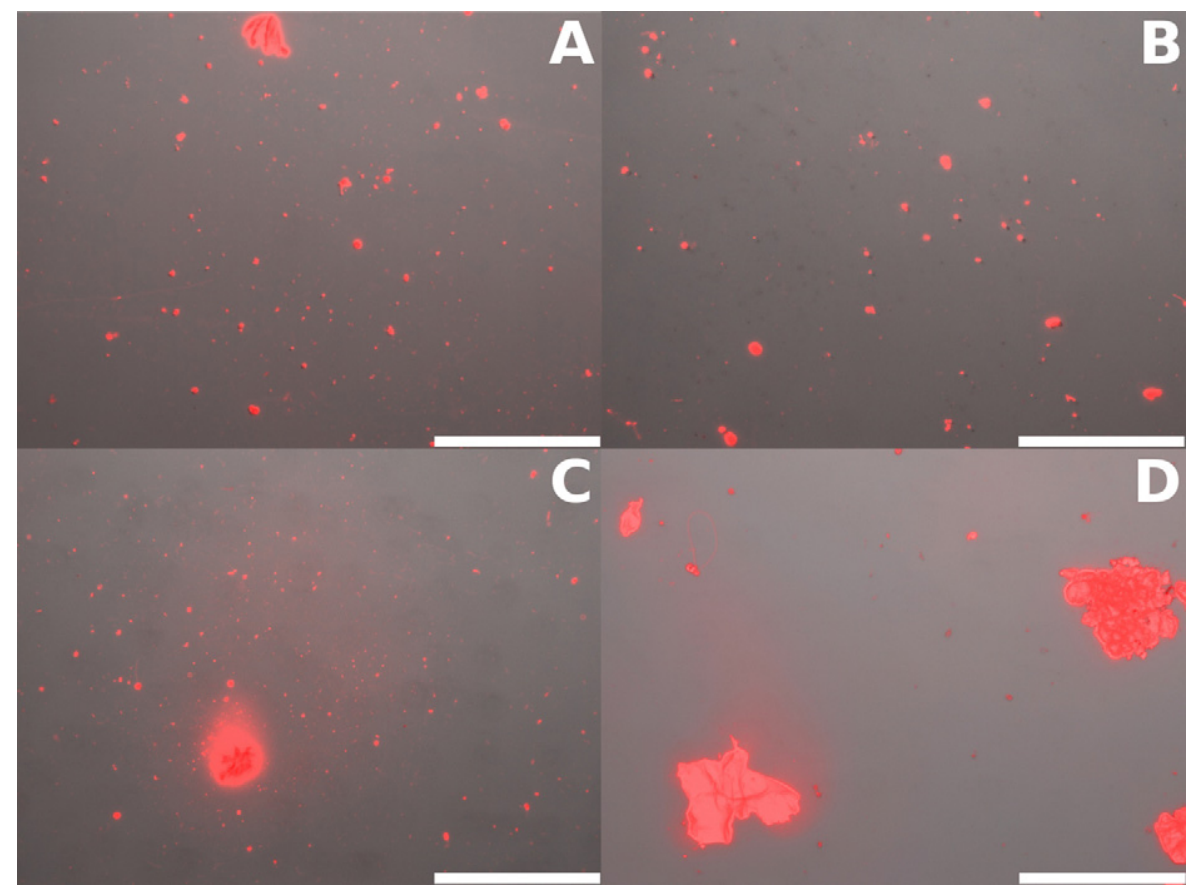

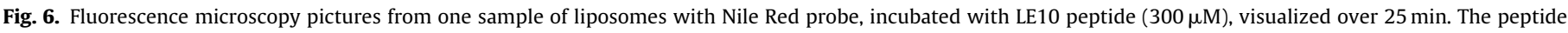

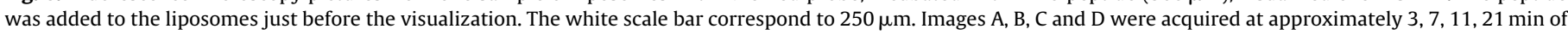
incubation. The pictures were acquired at different fields of view, due to quick quenching of the Nile Red fluorescence. 


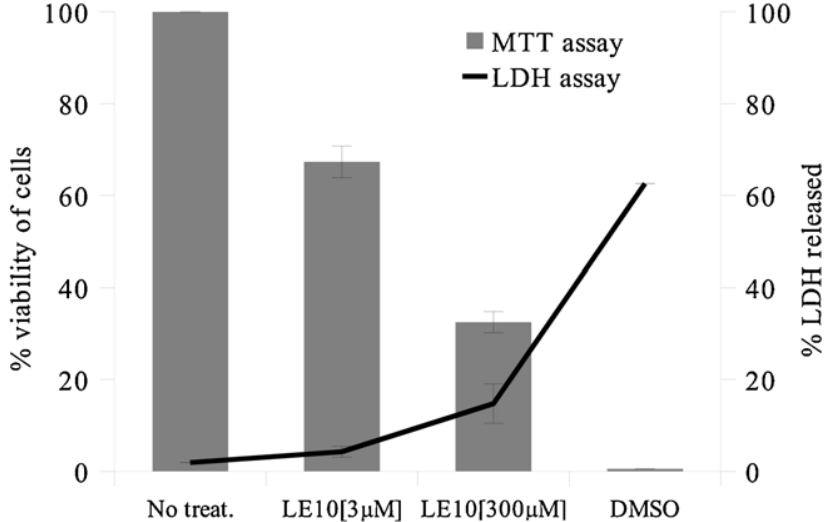

Fig. 7. Graphical representation of the results obtained in the cell assays, LDH (line) and MTT (columns), performed in the human cell-line BJ-5ta. Average values from three replicas for each condition (the error bars represent the standard deviation of the replicas at $95 \%)$.

visible. The bigger structures observed have red fluorescence, so these structures were composed by lipids and may in fact be huge lipidic films (see the big structures in Fig. 6D) and random lipid clusters. These results, together with those from DLS and fluorescence, point toward a global disruption of liposomes of EPC and CHOL by peptide LE10 at high concentration supporting the possibility that the mechanism observed in simulations is responsible for the disruption.

\section{In vitro assays}

To evaluate its activity in membranes and as mammalian cell killer, preliminary tests were performed with a human fibroblast cell line (BJ-5ta) exposed to LE10 peptide. The MTT (3-(4,5-dimethylthiazol-2-yl)-2,5-diphenyltetrazolium bromide) reduction assay, which mirrors the activity of vital metabolic enzymes present in mitochondrial membranes, and the LDH (lactate dehydrogenase) leakage assay, which is an indicator of cell membrane integrity, were chosen to study the eventual cytotoxic effect of LE10 on mammalian cells. The results are show in Fig. 7. For this cell line, the LE10 peptide showed interaction with the cell membrane, lightly compromising its integrity, since the release of the cytosolic enzyme LDH was approximately $4 \%$ and $15 \%$ for the incubation with the peptide at 3 and $300 \mu \mathrm{M}$, respectively. In turn, the toxicity of this peptide toward the metabolism of BJ-5ta cells has revealed itself high, as the percentage of viable cells after $24 \mathrm{~h}$ of incubation with LE10 decreased to $67 \%$ and $32 \%$ for 3 and $300 \mu \mathrm{M}$ of peptide, respectively. These results show that the LE10 peptide is toxic, even at low concentrations, to mammalian cells, suggesting that the mechanism of toxicity is not only through the disrupting of the plasmatic membrane. Some authors that studied the toxicity of AMPs in cancer cell-lines, proposed mechanisms of cell kill, other than the membrane disruption/destabilization, like mitochondria disintegration [37], changes in caspase activity, mitochondrial potential, reactive oxygen species production, and others [38].

The results of these cell assays cannot be directly compared with the results of molecular dynamics simulations, since the cell membrane is much more complex than the membrane model simulated. Nevertheless POPC is a zwitterionic lipid, the most common type of lipid in eukaryotic cells [39], and CHOL was present in a proportion similar to the proportions of this lipid in mammalian cells [39], making the simulation model a good approximation to study these mechanisms. The mechanism of internalization of the peptide via vesicles, suggested by the simulations, may be the same that leads to the results of LDH and MTT assays, since they show that the membrane was lightly disrupted. This disruption can be caused by the internalization of LE10 possibly by endocytosis. The increase on the amount of peptides that interact with the membrane can provoke high destabilization of the lipidic bilayer, which is in accordance with the results obtained with liposomes. The toxicity of peptide can be due to their propensity to interact with lipidic membranes (can interact with mitochondrial membrane, where, in fact, the reaction behind the MTT test takes place) already observed in simulations.

Although the performed experiments clearly show that LE10 promotes the membrane destabilization, liposome disruption and cell dead in the studied membranes, antimicrobial tests performed with six different microorganisms do not show antimicrobial properties for the peptide (results do not showed). The microorganisms tested were four bacteria, the Gram-positive Bacillus subtilis and Staphylococcus aureus, the Gram-negative Pseudomonas aeruginosa and Escherichia and two yeasts, the Saccharomyces cerevisiae and the Candida albicans. Due to the strong membrane interactions of LE10 observed in the previous experiments, we had thought that this peptide could have high antimicrobial activity, which was not confirmed. This fact is likely caused by the differences in composition and structure of membranes and by the existence of the cell-wall in the microorganisms. The presence of anionic phospholipids or phosphate groups of lipopolysaccharides (for Gram-negative bacteria) or acidic polysaccharides, teichoic acids, and lipoteichoic acids (for Gram-positive bacteria) or phosphomannans, chitin chains and the layer of beta-1,3-glucan (for yeasts) provide a negative surface charge to the cell-wall, and to the membranes of these organisms [40]. The cell-wall, with its negative surface, provides a physical barrier covering the lipidic membrane that can be inaccessible to cross by the LE10. The negative charge may prevent the LE10 to contact the cell membrane of these organisms due electrostatic repulsions, and the constituents of the cell-wall may trap the peptide or block its crossing.

\section{Major remarks}

The results of molecular dynamics simulations showed that the LE10 peptide strongly interacts with POPC/CHOL bilayers. First the peptides are attracted to the membrane surface, in an electrostatic process, and then the bilayer bending engulfs the peptides, in a manner similar to an endocytosis mechanism. The observation of full endocytosis is not accessible in the time scales simulated. The classification of this peptide as AMP or CPP is controversial, just like the division of both classes. LE10 is a short peptide with 10 negative charges provided by their glutamic acids residues, so this peptide composition fits in a class of AMPs designated by Anionic Peptides (according the classes defined in one work of Brogden [10]). The few authors that studied AAMPs proposed one mode of action that involves the formation of cationic salt bridges with metal ions like $\mathrm{Zn}^{2+}[41]$, which is not the case of this peptide. The mechanism of action of LE10 observed in simulations, as well as the antimicrobial tests, points toward the classification of this peptide as CPP, since there are many examples of CPPs internalized by different types of endocytosis. In CPPs the positive net charge is described as one essential feature. However Martín and co-authors that designed the anionic SAP(E) starting from the cationic SAP [17], showed that the negatively charged designed peptide have the same behavior and the same method of action (lipid-raft caveolae-mediated endocytosis) of the initial CPP. These results, together with the results presented here for LE10, show that positive net charge may not be an essential feature for CPPs. Despite this, the results of DLS and fluorescence with liposomes presented in this paper indicate that the peptide disrupt the membrane with increasing peptide concentration, which is characteristic of AMPs. 
These controversial results bring more arguments for the recent discussion about the classification of AMPs and CPPs as different types of peptides or as one big and diversified group. This peptide, like others mentioned in the introduction, has ambiguous proprieties which make it difficult to classify. The results presented here are in accordance with the hypothesis of AMPs and CPPs belonging to one group, making the LE10 one membrane active peptide (MAP).

\section{Conclusion}

In this work we describe one small membrane active anionic peptide, which reveals features of two types of peptides, CPPs and AMPs. These characteristics were determined by molecular dynamics and confirmed by experimental studies, which corroborate the power of molecular modeling research. The simulations show that this peptide is engulfed by lipidic bilayers of POPC, with or without $\mathrm{CHOL}$, in a process similar to early stages of endocytosis. The experimental results are in accordance with the simulations results, and also showed that high concentrations of the peptide lead to membrane disruption. The peptide also compromises the cell membrane of fibroblasts, being ultimately toxic for these cells although it is not for the microorganisms tested. This peptide is negatively charged, but strongly interacts with lipidic membranes, indicating that positive net charge may not be an essential feature for MAPs. Due to the features of LE10, small modifications in their structure may lead to promising properties in the areas of antibiotics design, and cancer therapy. The exciting properties of LE10, here demonstrate, makes it also a very promising tool for drug delivery design.

\section{Acknowledgements}

We would like to thank Dr. Andreia Vasconcelos from Department of Textile Engineering of University of Minho, Portugal, for providing technical support for this work.

\section{Appendix A. Supplementary data}

Supplementary data associated with this article can be found, in the online version, at http://dx.doi.org/10.1016/j.colsurfb. 2013.01.050.

\section{References}

[1] M.Zorko, U. Langel, Cell-penetrating peptides: mechanism and kinetics of cargo delivery, Adv. Drug Delivery Rev. 57 (4) (2005) 529-545.

[2] N.A. Brooks, D.S. Pouniotis, C.-K. Tang, V. Apostolopoulos, G.A. Pietersz, Cellpenetrating peptides: application in vaccine delivery, Biochim. Biophys. Acta Rev. Cancer 1805 (1) (2010) 25-34.

[3] B.R. Liu, Y. Huang, J.G. Winiarz, H.-J. Chiang, H.-J. Lee, Intracellular delivery of quantum dots mediated by a histidine- and arginine-rich HR9 cell-penetrating peptide through the direct membrane translocation mechanism, Biomaterials 32 (13) (2011) 3520-3537.

[4] I.M. Kaplan, J.S. Wadia, S.F. Dowdy, Cationic TAT peptide transduction domain enters cells by macropinocytosis, J. Control. Release 102 (1) (2005) 247-253.

[5] J.P. Richard, K. Melikov, H. Brooks, P. Prevot, B. Lebleu, L.V. Chernomordik, Cellular uptake of unconjugated TAT peptide involves clathrin-dependent endocytosis and heparan sulfate receptors, J. Biol. Chem. 280 (15) (2005) 15300-15306.

[6] A. Fittipaldi, A. Ferrari, M.Zoppé, C. Arcangeli, V. Pellegrini, F. Beltram, M. Giacca, Cell membrane lipid rafts mediate caveolar endocytosis of HIV-1 Tat fusion proteins, J. Biol. Chem. 278 (36) (2003) 34141-34149.

[7] S. Calvet, Cell internalization of the third helix of the antennapedia homeodomain is receptor-independent, J. Biol. Chem. 271 (30) (1996) $18188-18193$.

[8] H.D. Herce, A.E. Garcia, Molecular dynamics simulations suggest a mechanism for translocation of the HIV-1 TAT peptide across lipid membranes, Proc. Natl. Acad. Sci. U.S.A. 104 (52) (2007) 20805-20810.

[9] H.G. Boman, Peptide antibiotics and their role in innate immunity, Annu. Rev. Immunol. 13 (1995) 61-92.
[10] K.A. Brogden, Antimicrobial peptides: pore formers or metabolic inhibitors in bacteria? Nat. Rev. Microbiol. 3 (3) (2005) 238-250.

[11] B.M. Peters, M.E. Shirtliff, M.A. Jabra-Rizk, Antimicrobial peptides: primeval molecules or future drugs? PLoS Pathog. 6 (10) (2010) e1001067.

[12] F. Harris, S.R. Dennison, D.A. Phoenix, Anionic antimicrobial peptides from eukaryotic organisms, Curr. Protein Pept. Sci. 10 (6) (2009) 585-606.

[13] D. Rapaport, Y. Shai, Interaction of fluorescently labeled pardaxin and its analogues with lipid bilayers, J. Biol. Chem. 266 (35) (1991) 23769-23775.

[14] S.J. Ludtke, K. He, W.T. Heller, T.A. Harroun, L. Yang, H.W. Huang, Membrane pores induced by magainin, Biochemistry 35 (43) (1996) 13723-13728.

[15] E. Gazit, I.R. Miller, P.C. Biggin, M.S. Sansom, Y. Shai, Structure and orientation of the mammalian antibacterial peptide cecropin P1 within phospholipid membranes, J. Mol. Biol. 258 (5) (1996) 860-870.

[16] B. Bechinger, K. Lohner, Detergent-like actions of linear amphipathic cationic antimicrobial peptides, Biochim. Biophys. Acta Biomembr. 1758 (9) (2006) 1529-1539.

[17] I. Martín, M. Teixidó, E. Giralt, Design, synthesis and characterization of a new anionic cell-penetrating peptide: SAP(E), Chembiochem 12 (6)(2011) 896-903.

[18] K. Splith, I. Neundorf, Antimicrobial peptides with cell-penetrating peptide properties and vice versa, Eur. Biophys. J. 40 (4) (2011) 387-397.

[19] S.T. Henriques, M.N. Melo, M.A.R.B. Castanho, Cell-penetrating peptides and antimicrobial peptides: how different are they? Biochem. J. 399 (1) (2006) 1-7.

[20] S. Bobone, A. Piazzon, B. Orioni, J.Z. Pedersen, Y.H. Nan, K.-S. Hahm, S.Y. Shin, L. Stella, The thin line between cell-penetrating and antimicrobial peptides: the case of Pep-1 and Pep-1-K, J. Pept. Sci. 17 (5) (2011) 335-341.

[21] H.J. Jung, Y. Park, K.-S. Hahm, D.G. Lee, Biological activity of Tat (47-58) peptide on human pathogenic fungi, Biochem. Biophys. Res. Commun. 345 (1) (2006) 222-228.

[22] J. Jun, K. Jeong, Effective antibacterial action of Tat (47-58) by increased uptake into bacterial cells in the presence of trypsin, J Microbiol Biotechnol. 18 (5) (2008) 990-996.

[23] W.L. Zhu, S.Y. Shin, Effects of dimerization of the cell-penetrating peptide Tat analog on antimicrobial activity and mechanism of bactericidal action, J. Pept. Sci. 15 (5) (2009) 345-352.

[24] W.L. Zhu, S.Y. Shin, Antimicrobial, Cytolytic activities and plausible mode of bactericidal action of the cell penetrating peptide penetratin and its lys-linked two-stranded peptide, Chem. Biol. Drug Des. 73 (2) (2009) 209-215.

[25] C. Palm, S. Netzereab, M. Hällbrink, Quantitatively determined uptake of cell-penetrating peptides in non-mammalian cells with an evaluation of degradation and antimicrobial effects, Peptides 27 (7) (2006) 1710-1716.

[26] K. Sadler, K.D. Eom, J.-L. Yang, Y. Dimitrova, J.P. Tam, Translocating proline-rich peptides from the antimicrobial peptide bactenecin $7 \dagger$, Biochemistry 41 (48) (2002) 14150-14157.

[27] L.L.C. Schrödinger, The PyMOL Molecular Graphics System, Version 1.1, (2010).

[28] B. Sommer, T. Dingersen, C. Gamroth, S.E. Schneider, S. Rubert, J. Kruüger, K.-J. Dietz, CELLmicrocosmos 2.2 membrane editor: a modular interactive shapebased software approach to solve heterogeneous membrane packing problems, J. Chem. Inf. Model. (2011) 1165-1182.

[29] B. Hess, C. Kutzner, D. van der Spoel, E. Lindahl, GROMACS 4: algorithms for highly efficient, load-balanced, and scalable molecular simulation, J. Chem. Theory Comput. 4 (3) (2008) 435-447.

[30] S.-W. Chiu, S.A. Pandit, H.L. Scott, E. Jakobsson, An improved united atom force field for simulation of mixed lipid bilayers, J. Phys. Chem. B 113 (9) (2009) 2748-2763.

[31] Zetasizer Nano Series User Manual, Man 0317 Issue 1.1 Feb. 2004, England, Malvern Instruments Ltd., 2004.

[32] L. Miao, U. Seifert, M. Wortis, H.-G. Döbereiner, Budding transitions of fluidbilayer vesicles: the effect of area-difference elasticity, Phys. Rev. E: Stat. Nonlinear Soft Matter Phys. 49 (6) (1994) 5389-5407.

[33] H.-J. Woo, A. Wallqvist, Spontaneous buckling of lipid bilayer and vesicle budding induced by antimicrobial peptide magainin 2: a coarse-grained simulation study, J. Phys. Chem. B 115 (25) (2011) 8122-8129.

[34] J. Henriksen, A.C. Rowat, E. Brief, Y.W. Hsueh, J.L. Thewalt, M.J. Zuckermann, J.H. Ipsen, Universal behavior of membranes with sterols, Biophys. J. 90 (5) (2006) 1639-1649.

[35] P. Greenspan, S.D. Fowler, Spectrofluorometric studies of the lipid probe, nile red, J. Lipid Res. 26 (7) (1985) 781-789.

[36] M.C.a. Stuart, J.C. van de Pas, J.B.F.N. Engberts, The use of Nile Red to monitor the aggregation behavior in ternary surfactant-water-organic solvent systems, J. Phys. Org. Chem. 18 (9) (2005) 929-934.

[37] J. Slaninová, V. Mlsová, H. Kroupová, L. Alán, T. Tůmová, L. Monincová, L. Borovičková, V. Fučík, V. Ceřovský, Toxicity study of antimicrobial peptides from wild bee venom and their analogs toward mammalian normal and cancer cells, Peptides 33 (1) (2012) 18-26.

[38] S. Ghavami, A. Asoodeh, T. Klonisch, A.J. Halayko, K. Kadkhoda, T.J. Kroczak, S.B. Gibson, E.P. Booy, H. Naderi-Manesh, M. Los, Brevinin-2R(1) semi-selectively kills cancer cells by a distinct mechanism, which involves the lysosomalmitochondrial death pathway, J. Cell. Mol. Med. 12 (3) (2008) 1005-1022.

[39] B. Alberts, A. Johnson, J. Lewis, M. Raff, K. Roberts, P. Walter, Molecular Biology of the Cell, 5 th ed., Garland Science, New York, 2008, p. 624.

[40] V. Teixeira, M.J. Feio, M. Bastos, Role of lipids in the interaction of antimicrobial peptides with membranes, Prog. Lipid Res. 51 (2) (2012) 149-177.

[41] K.A. Brogden, M. Ackermann, P.B. McCray, B.F. Tack, Antimicrobial peptides in animals and their role in host defences, Int. J. Antimicrob. Agents 22 (5) (2003) 465-478. 